\title{
Communication skills training and assessment of food animal production medicine veterinarians: A component of a voluntary Johne's disease control program
}

\author{
Karen A. MacDonald-Phillips, ${ }^{1 *}$ () Shawn L. B. McKenna, ${ }^{1} \odot$ Darcy H. Shaw, ${ }^{2}$ Greg P. Keefe, ${ }^{1}{ }^{\oplus}$ \\ John VanLeeuwen, ${ }^{1}$ (D) Elpida Artemiou, ${ }^{3}$ and Cindy L. Adams ${ }^{4}$ (D) \\ ${ }^{1}$ Department of Health Management, Atlantic Veterinary College, University of Prince Edward Island, 550 University Avenue, Charlottetown, \\ Prince Edward Island, Canada C1A 4P3 \\ ${ }^{2}$ Department of Companion Animals, Atlantic Veterinary College, University of Prince Edward Island, 550 University Avenue, Charlottetown, \\ Prince Edward Island, Canada C1A 4P3 \\ ${ }^{3}$ Ross University School of Veterinary Medicine, West Farm, Basseterre, PO Box 334, St. Kitts and Nevis \\ ${ }^{4}$ Department of Veterinary Clinical and Diagnostic Services, University of Calgary, Veterinary Medicine, 1187785 St. NW, Calgary, AB, \\ Canada T3R 1J3
}

\section{ABSTRACT}

In food animal production medicine (FAPM), the success of control programs for infectious diseases that have serious animal health and economic consequences frequently rely on the veterinarian's effective communication and producer adherence to veterinary recommendations. However, little research has been conducted on communication skills of practicing FAPM veterinarians. During this study, we developed a communication training workshop intervention to support the Atlantic Johne's Disease Initiative. Seventeen FAPM veterinarians across 10 clinics practicing within Maritime Canada participated in a pre-post intervention study design. Communication skills were evaluated utilizing 3 assessment tools; an objective structured clinical exam (OSCE), standardized client feedback, and an instrument designed for veterinary participants to assess their self-efficacy. Study results showed that before training, communication skills of participating veterinarians had limitations, including skill deficits in communication tasks strongly associated with increased adherence to veterinary recommendations. Based on the 3 assessment tools, communication skills of participating veterinarians improved with the training provided. Significant increases were detected in pre- to postintervention self-efficacy percentage scores, OSCE percentage and global scores from expert raters, and OSCE percentage and global scores from standardized client feedback. These improvements emphasize the

Received April 28, 2021

Accepted October 13, 2021.

*Corresponding author: kamphillips@hotmail.ca importance of communication skills training specific to FAPM.

Key words: communication, communication skills assessment, communication skills training, food animal production medicine veterinarian, Johne's disease

\section{INTRODUCTION}

Johne's disease (JD), also known as paratuberculosis, is an incurable, chronic, infectious enteritis of domestic and wild ruminants caused by the bacterium Mycobacterium avium ssp. paratuberculosis and is one of the top disease priorities of Canadian dairy farmers, university researchers, and practicing veterinarians (Bauman et al., 2016). In an effort to reduce the prevalence and impact of Mycobacterium avium ssp. paratuberculosis infection in Atlantic Canada, the Atlantic Johne's Disease Initiative (AJDI), a management-based risk control program, was implemented from 2011 to 2014. Seventy percent of dairy herds in Atlantic Canada participated. The program relied on use of farm-specific risk assessments, conducted by AJDI-trained veterinarians. High-risk management practices were identified and modified to disrupt the Mycobacterium avium ssp. paratuberculosis infection cycle (Sorge et al., 2010a).

The ultimate success of a management-based control program is determined by adherence of producers to suggested changes in management practices (Sorge et al., 2010b). Previous studies on other JD control programs indicated that recommended management changes are not always being followed, particularly with increasing duration of participation in a control program (Wraight et al., 2000; Sorge et al., 2010a; Wolf et al., 2015). These studies, as well as studies on other infectious disease control programs, suggest that inad- 
equate veterinary communication likely contributed to poor adherence, and that improving the communication effectiveness of veterinarians could be important for success of future control programs (Jansen et al., 2010; Sorge et al., 2010a; Lam et al., 2011).

Skilled communication is a requisite to the practice of effective and compassionate veterinary medicine (Adams and Kurtz, 2017). It is considered a core clinical competency by the American Veterinary Medical Association Council on Education (American Veterinary Medical Association, 2020) as well as the Association of Veterinary Medical Colleges (Association of American Veterinary Medical Colleges, 2018) and the World Organization for Animal Health (World Organization for Animal Health, 2012). Research on, and teaching of, communication as an essential clinical skill in veterinary medicine is a growing discipline (Kurtz, 2006; Silverman et al., 2013; Adams and Kurtz., 2017). There is an expanding body of evidence in veterinary medical literature to demonstrate that communication competence is related to more efficient and satisfying consultations and improved outcomes of care, specifically satisfaction and adherence (Shaw et al., 2012; Adams and Kurtz, 2017; Ritter et al., 2019). Unfortunately, evidence also showed that communication deficiencies are evident during veterinary school, and that following graduation, learning communication skills on the job is not sufficient to master the necessary skills (Humphris, 2002; Shaw et al., 2004; Mullan and Kothe, 2010). Fortunately, training in communication has resulted in communication skill acquisition for veterinary students and companion animal practitioners (American Animal Hospital Association, 2009; Shaw et al., 2010; Adams and Kurtz, 2017). Veterinary schools around the globe are incorporating communication skills education into their curriculum by training faculty and developing programs that use lectures, small-group interactive sessions involving simulated clients and facilitators, video feedback, assigned readings, role-play and web-based programs (Hargie et al., 2010; Artemiou et al., 2013; Shaw, 2019).

Studies in food animal production medicine (FAPM) suggest that veterinary practitioners' communication skills can benefit from additional training (Cipolla and Zecconi, 2015; Ritter et al., 2018; Ritter et al., 2019). Petrovski and McArthur (2015) described specific communication steps to facilitate veterinary-client consultations in bovine medicine, both at the individual animal and population level. These steps were based on a modified Calgary-Cambridge guide (CCG), a comprehensive set of communication skills initially developed for human medicine (Silverman et al., 2013) and then modified for veterinary medicine (Adams and Kurtz, 2006, 2017; Radford et al., 2006). However, there have been no reports of education curricula for communication skills being developed, implemented, or assessed specifically for practicing FAPM veterinarians.

Various methods exist for assessing communication skills (Shaw, 2019). Objective structured clinical examination (OSCE) offers a format for assessing communication skills of undergraduate health professional students that is valid for clinical skills, not just knowledge, and has high reliability (Davis et al., 2006; Hodges, 2006). Objective structured clinical examination provides a simulated environment where students can demonstrate their abilities across various contexts following a standardized examination format (Hecker et al., 2012). During the OSCE, the learner interacts with standardized clients (SC), who are trained to portray a client's concerns in a standardized manner (Adams and Ladner, 2004; Barton et al., 2006; Artemiou et al., 2014a).

Self-efficacy questionnaires, which represent an individual's possibly biased self-evaluation on successfully performing a specified task, such as communication skills, is another tool that has frequently been used in human medicine (Mullan and Kothe, 2010; Roter et al., 2012; Gulbrandsen et al., 2013). A variety of internal and external factors (e.g., personal knowledge and skills, self-esteem, task complexity, and stress) can influence a person's judgment of self-efficacy (Ammentorp et al., 2007). Use of self-efficacy assessment in conjunction with outcome measures, such as an OSCE, has not been reported in veterinary communication skills training.

In this study, a communication skills training workshop and pre-post assessments of communication skills were conducted with FAPM veterinarians as part of the AJDI. It was hypothesized that training in communication would lead to communication skill improvement for the FAPM veterinarians. Accordingly, the goals of the study were to (1) assess communication skills of veterinarians before communication skills training using 3 assessment tools: OSCE, SC feedback, and selfefficacy; (2) develop and implement a communication skills training workshop for FAPM veterinarians; and (3) assess the effect of a communication skills training workshop on communication skills of veterinarians using the same 3 assessment tools. The results were intended to deepen understanding of how to teach and assess communication skills for FAPM veterinarians who are part of an infectious disease control program. Additionally, our study results would inform development of communication skills training and assessment 
specific to FAPM at the Atlantic Veterinary College (AVC), University of Prince Edward Island.

\section{MATERIALS AND METHODS}

Study implementation is described in the following sections, and the layout of its component parts is shown in Figure 1.

\section{Participants, SC, Coaches, and Raters}

Email and telephone invitations to the communication skills workshop were sent to $48 \mathrm{FAPM}$ veterinarians trained through the AJDI across 18 veterinary clinics practicing within Maritime Canada. Seventeen of the 48 veterinarians agreed to participate. A sample size calculation was not performed before commencement of the study, but resources were available and workshop design was ideal for 20 participants; as such, 3 AVC senior veterinary students who had received specialized training in FAPM also partook in the training opportunity. This manuscript is focused on the practicing FAPM veterinarians only. Participant written consent was obtained to allow video recording of the OSCE for research purposes at the start of the workshop (see Supplemental File S1; https://data.upei.ca/islandora/ object/researchdata\%3A680; MacDonald-Phillips et al., 2021) and verbal consent was obtained for undergoing training and assessment when participants volunteered to function as certified AJDI veterinarians. Animal Use Protocols (Protocol 11-038 file number 6004374) and Biosafety Permits (file number 6004379) were approved by their respective compliance bodies for this project. Based on the information in those protocols submitted at that time in 2010, research ethics board approval was deemed to be not necessary by the University of Prince Edward Island.

Eight retired dairy producers from Prince Edward Island were recruited to be SC and trained by an experienced SC trainer [B. Gromoff, consulting standardized client coordinator at University of Calgary Veterinary Medicine (UCVM)]. All 8 SC participated in OSCE and $5 \mathrm{SC}$ supported small-group communication training sessions.

Six coaches were recruited to facilitate small-group experiential communication training sessions. Four coaches were veterinarians from the Department of Health Management of AVC who had extensive experience with FAPM ( 3 faculty members and 1 graduate student). These coaches participated in small-group facilitation training before the communication skills training workshop. The other 2 coaches had substan- tial expertise in experiential teaching of communication skills (C. Adams, professor at UCVM who directs the clinical communication program, and J. Wilson, a veterinarian with extensive coaching experience in the communication program at UCVM).

Four raters were recruited to independently review all OSCE video recordings, blinded to OSCE type (i.e., pre- vs. postintervention OSCE). Two raters were veterinary students enrolled at AVC who completed their first and second years of the 4-yr program and had experience with FAPM. Before rating, the students participated in a training session designed to enhance inter- and intrarater reliability. Student raters were referred to as "trained raters." The other 2 raters were considered experts based on their experience in communication skills assessment, including rating communication OSCE (referred to as "expert raters"; C. Adams and E. Artemiou, associate professor of clinical communication at Ross University School of Veterinary Medicine). For conciseness, this manuscript is focused on expert raters only. Comparison of trained raters and expert raters will be the focus of a future manuscript.

\section{Preparations for the Communication Skills Workshop Intervention}

The framework used to develop the intervention was the CCG. The CCG is comprised of 73 evidence-based communication process skills and organizes the medical consultation into a framework of 6 communication domains and objectives (Adams and Kurtz, 2012; Silverman et al., 2013). Participants were expected to learn and demonstrate skills associated with each of the following CCG domains: (1) initiating the session, (2) gathering information, (3) providing structure, (4) building the relationship, (5) explaining and planning, and (6) closing the session.

Pre- and postintervention OSCE cases were designed to assess the participants' communication skills. OSCE cases representing real-life FAPM scenarios were developed, adapted and standardized by FAPM veterinarians at the AVC. Likewise, real-life FAPM cases were also developed, tested and standardized at UCVM for coach-facilitated, small-group training sessions, to allow participants to practice skills through interactions with SC.

For self-efficacy assessment tools, questionnaires were adapted from AVC Client Communication Rotation Surveys. For communication assessment using SC feedback, a SC feedback form was adapted from the UCVM clinical communication skills program. To rate the participant's communication skills based on the 

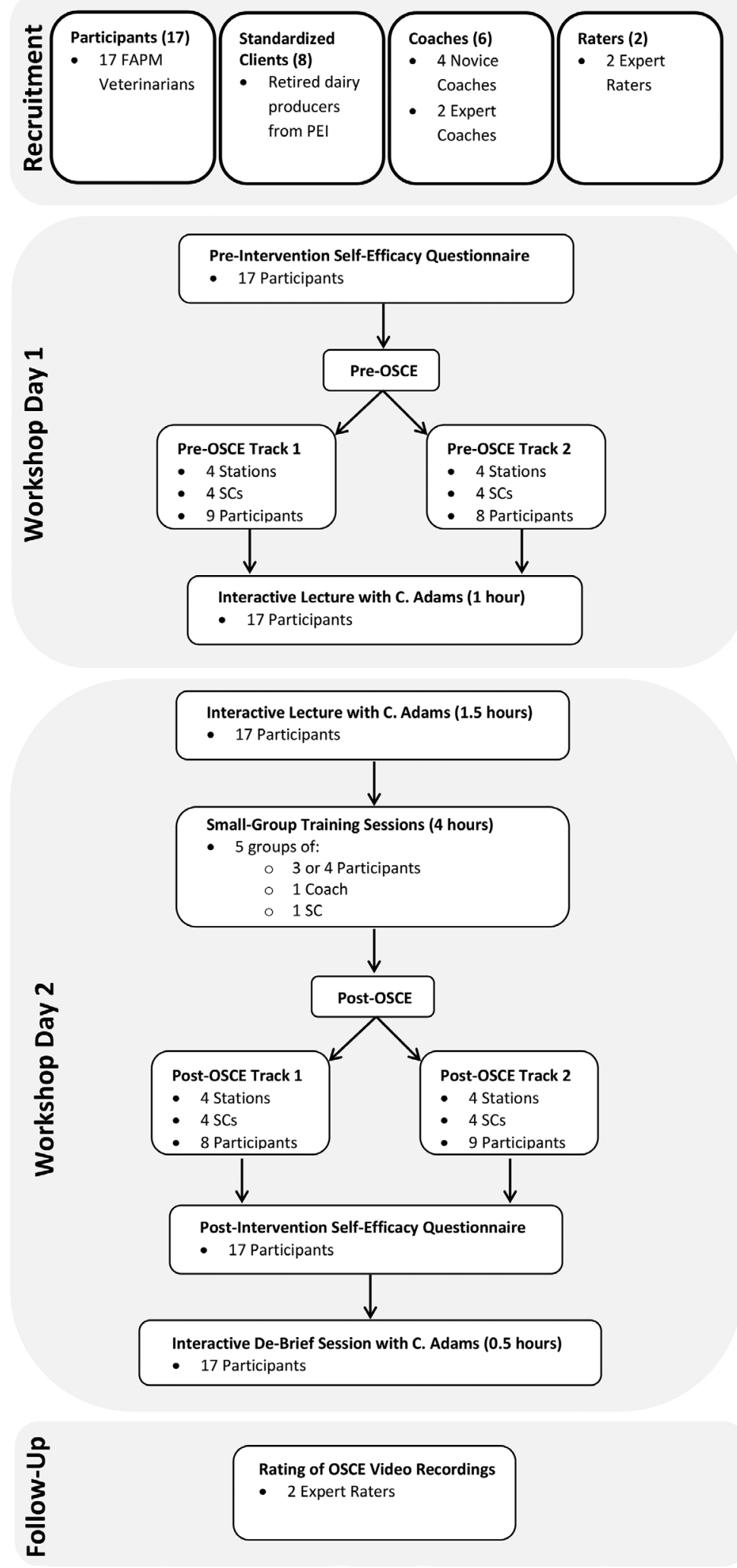

Figure 1. Layout of the communication skills training and assessment workshop for 17 food animal production medicine (FAPM) veterinary participants, 8 standardized clients (SC), 6 coaches, and 2 expert raters, in Atlantic Canada in 2014. PEI = Prince Edward Island; OSCE = objective structured clinical exam. Lecture by C. Adams, professor at University of Calgary Veterinary Medicine, who directs the clinical communication program. 
CCG, an OSCE checklist was developed from the CCG and contained communication skills for each of the 6 communication domains.

\section{Implementation of the Communication Skills Training Workshop Intervention}

The communication skills training workshop was held in the winter of 2014 over $1.5 \mathrm{~d}$ ( $13 \mathrm{~h}$ in total). In human medicine, training programs were found to improve communication skills if they lasted for at least $1 \mathrm{~d}$ (Berkhof et al., 2011).

All participants completed a self-efficacy questionnaire (Figure 1) before the communication skills training intervention (see Supplemental File S2; https:// data.upei.ca/islandora/object/researchdata\%3A680; MacDonald-Phillips et al., 2021). Participants scored 13 self-efficacy items using a 5 -point Likert scale, with 5 being "excellent" and 1 being "poor." Items were totaled and converted into a percentage of self-efficacy items (Ammentorp et al., 2007; Gulbrandsen et al., 2013). The pre-intervention survey also included open-ended questions inquiring about participant's expectations for the communication skills training workshop.

Additionally, all participants completed a pre-intervention OSCE that consisted of 4 stations (Figure 1). For time efficiency, 2 OSCE tracks were run simultaneously with the same 4 cases being portrayed in each track. Participants interacted with one SC portraying a specific case at each station. Before entering a station, participants had 2 min to review the OSCE task sheet. An example task sheet used in the OSCE is shown in Supplemental File S3 (https://data.upei.ca/islandora/ object/researchdata\%3A680; MacDonald-Phillips et al., 2021). Participants were informed that they were being assessed on communication skills and not medical knowledge. Participants had $10 \mathrm{~min}$ to complete their interaction with the SC. A 2-min verbal warning was given before the end of each consultation. Interactions between participants and SC were video-recorded at each station. Five support personnel assisted with OSCE set-up, timing, and movement of participants through stations.

Following each OSCE consultation, the SC completed an SC feedback form on the participant's communication performance (see Supplemental File S4; https:// data.upei.ca/islandora/object/researchdata\%3A680; MacDonald-Phillips et al., 2021). For each of the 17 items in the SC feedback form, SC indicated if the item skill was demonstrated by choosing "no," "yes but" or "yes." The "yes but" score indicated partial demonstration of skill. Feedback item ratings were converted to a score of 0 for "no," 1 for "yes but" and 2 for "yes."
Scores were totaled and converted to a percentage of feedback items (Artemiou et al., 2014a).

For each participant, SC also indicated if they would choose that person as their veterinarian (a global feedback rating), by choosing "no," "yes but" and "yes." This global feedback rating was also converted to a score of 0 for "no," 1 for "yes but" and 2 for "yes."

The intervention (Figure 1) included 2 interactive lectures $(1 \mathrm{~h}$ on the first day and $1.5 \mathrm{~h}$ on the second day). These lectures were presented by a member of the faculty of UCVM with substantial experience in teaching communication skills (C. Adams).

Small-group training sessions followed the lectures, where participants were divided into 5 groups. Each group also included a coach and a SC. During training sessions, each participant spent a minimum of $45 \mathrm{~min}$ practicing their communication skills with a $\mathrm{SC}$ and received individualized feedback and active coaching from coaches. Following the interaction, small-group members, coaches and SC provided feedback and the opportunity for the interviewing participant to return to their interaction with the SC to integrate feedback.

Postintervention communication skills assessments were conducted following small-group training sessions (Figure 1). These assessments were similar to preintervention assessment, with all participants completing a self-efficacy questionnaire and an OSCE. Open questions about participants' satisfaction with the workshop and 11 self-efficacy items were included in the postintervention survey (see Supplemental File S5; https://data.upei.ca/islandora/object/researchdata \%3A680; MacDonald-Phillips et al., 2021). Different OSCE cases were used for postintervention OSCE assessments. Five support personnel again assisted with postintervention OSCE set-up, timing, and movement of participants through stations. Following postintervention assessment, there was a 30-min debrief session with veterinary participants, study design team, and coaches.

Following the workshop, all OSCE video recordings were rated by all raters. Raters were unaware of OSCE type (whether it was pre- or postintervention) and used an OSCE checklist (see Supplemental File S6; https:/ /data.upei.ca/islandora/object/researchdata\%3A680; MacDonald-Phillips et al., 2021) to score how the 22 communication process skills were demonstrated by choosing "no," "yes but" or "yes." The "yes but" score indicated that the skill was partially demonstrated. The checklist item ratings were converted to a score of 0 for "no," 1 for "yes but" and 2 for "yes." Checklist item scores were totaled per OSCE consultation for each rater and converted to a percentage of the checklist items (Hecker et al., 2012). Furthermore, rat- 
ers gave a global rating score for participants' overall communication performance per case using a 5-point Likert scale, with 5 being "excellent" and 1 being "poor."

\section{Statistical Analyses}

Missing data frequency and distribution were assessed to determine the randomness of the missing data. Due to the small number of participants, where the missing data were considered random, they were addressed using several different methods. Person-mean imputation (Eekhout et al., 2014) was used for missing items in self-efficacy questionnaires, OSCE checklists, and SC feedback forms (missing items were imputed using the mean of participants' nonmissing items). For comparison purposes, multiple imputation (Eekhout et al., 2014) for missing items in OSCE checklists was also performed to compare the mean percentage scores and $95 \%$ confidence intervals (CI) with those achieved using person-mean imputation. Available-case analysis (van Ginkel et al., 2010), using all cases which have observed values on variables that are part of the analyses, was used for global scores in both the OSCE checklists and the SC feedback forms because these scores did not have other similar items to assist with imputation.

Statistical analyses were performed using STATA/IC 12.1 (StataCorp LP) for the 17 participants who were practicing FAPM veterinarians. Significance was set at a $P<0.05$. Descriptive statistics (means and 95\% CI) were calculated for participant data, self-efficacy questionnaires and OSCE, in total and stratified by OSCE type. Paired $t$-tests were used to confirm whether scores were significantly higher postintervention compared with pre-intervention (self-efficacy percentage, OSCE checklist percentage, OSCE global checklist score, SC feedback percentage, and SC global feedback score). Reliability, inter-rater agreement and variance components for all main and interaction OSCE effects were assessed using generalizability theory (data not shown). The OSCE checklist percentage and global checklist scores used in the statistical analyses in this current manuscript were from the expert raters only. Openended questions in self-efficacy questionnaires were analyzed by identifying patterns of themes based upon frequency distributions of participant responses.

\section{RESULTS}

\section{Participants}

Proportions of veterinarians and clinics who participated in this study were $35 \%$ (17 of 48 invitees) and
$56 \%$ (10 of 18 clinics), respectively. Sixty-five percent $(\mathrm{n}=11)$ of participants self-identified as female and $35 \%(\mathrm{n}=6)$ self-identified as male. The median years since graduation with their doctor of veterinary medicine degree was 12 (range: 1-29).

\section{Missing Data}

Self-efficacy questionnaires had $0.5 \%$ of items missing. Overall, $6 \%$ of questionnaires $(n=2)$ had incomplete data, and the mean percent of missing items per incomplete questionnaire was $9 \%$. Based on dissimilar questions being unanswered, the missing data were considered missing completely at random (MCAR). Person-mean imputation was used to replace missing self-efficacy questionnaire items.

During the OSCE, camera malfunction at one station resulted in no video recording in 11 of 136 consultations $(8 \%)$. The OSCE checklists from recorded consultations had $2 \%$ of items missing from nonresponse that was considered MCAR. Overall, 22\% of OSCE checklists had incomplete data, and the mean percent of missing items per incomplete OSCE checklist was 8\% (95\% CI: $6,9 \%)$. Person-mean imputation was used to replace the missing checklist items for the analyses. Checklist percentage scores and 95\% CI that were calculated after using person-mean imputation were compared with scores calculated after using multiple imputation. The scores did not differ significantly, with means of $58.0 \%$ (95\% CI: $55.9,60.2 \%$ ) and $57.9 \%$ (95\% CI: 55.8 , $60.1 \%)$, respectively.

One global score was missing for the OSCE observations (0.4\%). With only 1 missing observation, that missing data were considered MCAR. Available-case analysis was used to analyze nonmissing OSCE global scores.

During OSCE, 15 SC feedback forms were misplaced and 3 were not filled out from 136 consultations (13\% of the SC feedback forms in total). Misplaced forms were primarily from one SC (13 of 15 forms). The 18 consultations with no SC feedback data were discarded from the data set. The remaining SC feedback forms had $5.1 \%$ of items missing due to nonresponse. Overall, $29 \%$ of forms had incomplete data and the mean percent of missing items per incomplete form was $18 \%$ (95\% CI: 13, 23\%). Based on dissimilar items being unanswered, the missing data were considered MCAR. Person-mean imputation was used to replace missing SC feedback form items.

The SC feedback forms had 20 global scores missing for the 118 consultations retained in the data set $(17 \%)$. Based on which scores remained unanswered, the missing data were considered MCAR. Similar to 
OSCE global scores, available-case analysis was used to analyze the SC global scores.

\section{Pre- and Postintervention Self-Efficacy Questionnaires}

On the pre-intervention self-efficacy questionnaire, $82 \%$ of participants reported that the primary reason for attending the communication skills training workshop was to improve their communication skills ( $\mathrm{n}=$ 14). Before the workshop, participants described their previous communication training as below average, with a mean of 1.8 out of 5 (95\% CI: $1.3,2.3)$. They also reported their knowledge of communication skills as fair, with a mean of 2.3 out of 5 (95\% CI: 1.8, 2.8).

Following the workshop, participants reported improvement of their knowledge surrounding communication skills, with a mean of 3.8 out of 5 (95\% CI: 3.6, 4.0) on the postintervention self-efficacy questionnaire. Suggested ways to improve the workshop included: lengthening the workshop $(\mathrm{n}=4)$ and spending more time on experiential practice $(\mathrm{n}=4)$.

A significant difference was detected between the pre- and postintervention self-efficacy percentages [65.7\% (95\% CI: $61.4,69.9 \%)$ and $73.4 \%$ (95\% CI: 70.7, $76.0 \%)$, respectively; paired $t$-test: $\mathrm{t}(32)=-3.9, P<$ $0.01]$.

\section{Pre- and Postintervention Objective Structured Clinical Exams}

Table 1 shows the pre- and postintervention OSCE descriptive statistics for percentage and global scores from expert raters and SC. Based on nonoverlapping 95\% CI, all percentage and global scores were significantly higher postintervention compared with preintervention.
Paired $t$-tests confirmed significant increases in percentage and global scores from pre- to postintervention OSCE from expert raters [paired $t$-test for percentage score: $\mathrm{t}(247)=-12.86, P<0.01$; paired $t$-test for global score: $\mathrm{t}(246)=-14.03, P<0.01]$. Based on paired $t$-tests of the SC feedback, there was also confirmation of significant increases in percentage and global scores from pre- to postintervention OSCE [percentage score paired $t$-test: $\mathrm{t}(116)=-3.93, P<0.01$; global score paired $t$-test: $\mathrm{t}(96)=-3.46, P<0.01]$.

Table 2 shows pre- and postintervention OSCE descriptive statistics for the task percentage scores for each of the 6 communication domains from expert raters. Based on nonoverlapping 95\% CI, all task percentage scores were significantly higher postintervention than pre-intervention. Paired $t$-tests confirmed significant increases in task percentage scores from pre- to postintervention OSCE for all 6 communication domains $(P<$ 0.01). The communication tasks with the highest and lowest percentage scores were "gathering information" and "closing the session," respectively.

\section{DISCUSSION}

This is the first study that followed an experimental, pre- and postintervention design to evaluate how a communication skills training workshop improved the communication skills of practicing FAPM veterinarians. Main findings of the study were (1) before training, communication skills of participants had limitations, including deficits in skills that have been strongly associated with adherence to veterinary recommendations; and (2) an experiential communication skills training workshop improved the communication skills of participants.

Before communication skills training, participants reported their knowledge of communication skills was

Table 1. Descriptive statistics of percentage scores and global scores for the 17 food animal production medicine veterinary participant pre- and postintervention objective structured clinical exams (OSCE), by 2 expert raters and 8 standardized clients (SC), in Atlantic Canada in 2014

\begin{tabular}{|c|c|c|c|c|}
\hline \multirow[b]{2}{*}{ Assessor } & \multicolumn{2}{|c|}{ Pre-intervention OSCE } & \multicolumn{2}{|c|}{ Postintervention OSCE } \\
\hline & Percentage score & Global score & Percentage score & Global score \\
\hline Mean & 47.4 & 1.7 & 69.1 & 3.3 \\
\hline $95 \% \mathrm{CI}$ & $45.3,49.5$ & $1.6,1.8$ & $66.4,71.7$ & $3.1,3.5$ \\
\hline No. of observations & 127 & 126 & 122 & 122 \\
\hline \multicolumn{5}{|l|}{$\mathrm{SC}^{2}$} \\
\hline No. of observations & 59 & 48 & 59 & 50 \\
\hline
\end{tabular}

${ }^{1} \mathrm{OSCE}$ checklist.

${ }^{2} \mathrm{SC}$ feedback form. 
only fair. This self-assessment was substantiated by low percentage and global scores from expert raters in the pre-intervention OSCE. Percentage scores indicated that the performance level of participants was less than half of what was expected to properly demonstrate the communication skills. The percentage scores were supported by global scores that were also only fair preintervention.

Communication deficiencies to this degree would translate into ineffective communication with participants' food animal production clients, including AJDI participants. Previous research has shown that specific communication tasks inherent to the CCG are strongly associated with adherence to recommendations, particularly in building the relationship and explanation and planning (Silverman et al., 2013). Participants' OSCE performances showed deficits in both of these domains. Insufficient communication skills specific to these 2 communication domains would likely have a negative effect on the adherence of participants' food animal production clients to recommendations, including AJDI participants. Reduced adherence of AJDI producers would result in unsatisfactory implementation of risk assessment and management plan recommendation, and undermine the goal of controlling JD in Atlantic Canada.
Communication skills of participants improved following training. This study clearly demonstrates that communication interventions such as face-to-face interactive lectures and small-group teaching were effective at helping FAPM veterinarians improve their communication skills. Improved performance was also observed for individual communication tasks, including building the relationship and explanation and planning. Intentional and effective use of communication skills specific to these 2 communication domains should translate to improved adherence of veterinarians' food animal production clients, including AJDI participants. Improved adherence of AJDI participants to risk assessment and management plan recommendations would ultimately support the goal of controlling JD in Atlantic Canada. This conclusion is supported by previous research which suggested that improved veterinary communication would lead to enhanced adoption of infectious disease control programs by dairy producers (Sorge et al., 2010a; Kleen et al., 2011; Lam et al., 2011).

To investigate the reliability of the 4-station communication OSCE and if improvements in OSCE checklist scores were predominately due to participant communication skill or other facets of the study design, analyses using generalizability theory were conducted and described in chapter 6 of the $\mathrm{PhD}$ thesis of the

Table 2. Descriptive statistics of task percentage scores for the 17 food animal production medicine veterinary participant pre- and postintervention objective structured clinical exams (OSCE) from 2 expert raters in Atlantic Canada in 2014

\begin{tabular}{lcc}
\hline Task & $\begin{array}{c}\text { Pre-intervention OSCE } \\
\text { task percentage score }\end{array}$ & $\begin{array}{c}\text { Postintervention OSCE } \\
\text { task percentage score }\end{array}$ \\
\hline $\begin{array}{l}\text { Initiating the session } \\
\text { Mean }\end{array}$ & 44.45 & 65.50 \\
95\% CI & $127.23,47.66$ & $61.71,69.29$ \\
No. of observations & 59.15 & 122 \\
Gathering information & $55.40,62.90$ & 80.07 \\
Mean & 127 & $76.80,83.33$ \\
$95 \%$ CI & 33.65 & 122 \\
No. of observations & $28.59,38.71$ & 57.51 \\
Providing structure & 127 & $51.67,63.35$ \\
Mean & 55.31 & 122 \\
95\% CI & $51.60,59.02$ & 73.60 \\
No. of observations & 127 & $69.56,77.64$ \\
Building the relationship & & 122 \\
Mean & 45.76 & 70.07 \\
95\% CI & $42.62,48.89$ & $66.22,73.92$ \\
No. of observations & 127 & 122 \\
Explanation and planning & & 47.54 \\
Mean & 25.09 & $40.96,54.12$ \\
95\% CI & $20.14,30.03$ & 122 \\
No. of observations & 127 & \\
Closing the session & & \\
Mean & & \\
95\% CI & No. of observations &
\end{tabular}


lead author (MacDonald-Phillips, 2021). Generalizability theory accounts for several sources of measurement error, such as error due to raters, OSCE station, and OSCE tract (Goodwin, 2001). For conciseness, this current manuscript focused on the objectives as outlined.

Alternate methods could have been used to investigate or reduce the likelihood that improvements in OSCE checklist scores were influenced by other facets of the study design; for example, using a crossover design for cases within track or a control group. To reduce the risk of differences in case difficulty between the pre- and postintervention OSCE affecting the outcomes, different cases could have been used in the pre-intervention OSCE tracks. For the postintervention OSCE, the cases could have been switched between OSCE tracks, allowing for the same cases to be used both pre- and postintervention, on different participants. Use of a control group, a group whose communication skills were assessed but did not participate in the communication skills training interventions, could have strengthened this study. In a pre-post OSCE study by Artemiou et al. (2013), findings indicated that participating in pre-intervention OSCE cases resulted in learning and improved comprehension and use of communication skills by control group participants. However, the participants in the control group scored lower mean differences between pre- and postintervention OSCE than did the intervention groups (Artemiou et al., 2013). The number of veterinary participants in this study precluded the use of a control group. Subsetting the study population into intervention and control groups would have led to limited power to detect differences between groups. In addition, in our experience, ethics requires the communication skills training components be rerun for the control group after communication skills assessments are complete. This was not feasible for our study. Requiring practicing FAPM veterinarians to go through the assessments as a control group and then dedicate further in-person time to do the experiential communication skills training components would have the reduced response rate. Further research into communication skills training for practicing FAPM veterinarians (e.g., using a larger study population, including a control group, using a crossover design for OSCE cases within track, evaluation of additional variables for association with improvement in scores following training) is recommended to further improve knowledge and understanding of the effect of training.

In this study, the relatively low veterinary participation rate could have introduced a study limitation of nonresponse bias. It is possible that AJDI-trained veterinarians who participated in the communication skills training workshop had more need or interest to improve their communication skills than their nonparticipating colleagues. This possibility is supported by the preintervention self-efficacy response of most participants that their primary reason to attend the workshop was to improve their communication skills. This bias could result from differences in their baseline communication skills and lead to differences in responses to communication training compared with nonparticipating colleagues.

Missing data bias may have been introduced by video recording failure. However, this bias would only apply if there was a difference in communication performance scores related to the station with the malfunctioning camera, which is unlikely. Missing items within the self-efficacy questionnaires, OSCE checklists, and SC feedback forms may also have introduced item missing data bias. It is unlikely that any of these missing data biases would be large as the pattern of missing data in each of these communication assessment tools was MCAR, and the percent of items missing was small.

Bias could also have been introduced into this study by use of person-mean imputation. However, for OSCE percentage scores, there was no difference detected between score estimates and the 95\% CI when comparing person-mean imputation and multiple imputation. Multiple imputation has been recommended to handle item score missing data; however, it is more complicated than simple imputation procedures, such as person-mean imputation. When only a small amount of item scores are missing $(<25 \%)$ in only a small amount of cases $(<10 \%)$, simple imputation methods may be preferred purely for practical reasons (Eekhout et al., 2014). The lack of differences when comparing the 2 imputation methods provided us with confidence that the simpler imputation method was appropriate.

Assessment tools were adapted from UCVM and AVC clinical communication skills programs and developed from the CCG for this study. Additional analyses to evaluate these tools further, such as factor component analysis or principal component analysis, would be of benefit.

In this study, the CCG was used as a framework for communication skills assessment and interventions with FAPM veterinarians. We found that the CCG's focus on, and organization of, the veterinary consultation applied well to the introduction and early communication learning for FAPM veterinarians in this workshop. This is partially supported by a study that modeled communication in production animal medicine (Kleen et al., 2011). That study agreed that the CCG model of the consultation process applies to companion animal medicine, as well as to most aspects of traditional, curative, farm animal practice. However, the authors suggested 
that in veterinary advisory practice, such as FAPM practice, the communication between veterinarian and owner goes beyond the medical curative consultation, and as such, additional communication instruments should be used (Kleen et al., 2011). In future, additional research into the most appropriate communication instrument for advanced training in communication for FAPM veterinarians, particularly FAPM veterinarians in advisory roles, should be considered.

The communication skills training workshop in this study was conducted in-person over $1.5 \mathrm{~d}$, and used interactive lectures and small-group training as communication interventions. Previous research has found that communication training programs improved communication skills if they lasted for at least $1 \mathrm{~d}$ (Berkhof et al., 2011) and that small-group training was the most effective approach for enhancing communication skills (Artemiou et al., 2013). However, the in-person time commitment required was a concern for some FAPM veterinarians. Some solo practitioners were unable to attend because they couldn't leave their practice for the length of the workshop, and multiveterinarian clinics could only send some, rather than all, veterinarians in the practice in order maintain clinic operations. These barriers for veterinary participation in the workshop may have introduced sampling bias into the study. Sampling bias could compound nonresponse bias described above, and in turn lead to further differences in responses to communication training compared with nonparticipating colleagues.

Participating veterinarians suggested that lengthening the workshop or allowing more time for communication training would have been beneficial. Considering the time constraints already experienced by FAPM veterinarians, an alternative could be to incorporate web-based communication interventions into a communication skills workshop. Research has found that web-based communication training was an effective communication training method (Roter et al., 2012; Artemiou et al., 2013, 2014b). Web-based communication training could be particularly useful to introduce communication theory and describe the necessary communication skills before the workshop. This would allow more time for participants to practice communication skills during the in-person workshop (using small-group training and video skill-spotting exercises), and would likely lead to less workshop fatigue.

\section{CONCLUSIONS}

Based on this study, development and implementation of communication skills training and assessment increases the communication competency of FAPM vet- erinarians. This training would be particularly relevant for those veterinarians involved in infectious disease control programs.

\section{ACKNOWLEDGMENTS}

The project was funded by the Dairy Farmers of Prince Edward Island (Charlottetown, PE, Canada), Nova Scotia (Truro, NS, Canada), New Brunswick (Sussex, NB, Canada), and Newfoundland and Labrador (St. John's, NL, Canada); and Agriculture and Agri-Food Canada (Ottawa, ON, Canada) in conjunction with the Provinces of Prince Edward Island (Charlottetown, PE, Canada), Nova Scotia (Halifax, NS, Canada), New Brunswick (Fredericton, NB, Canada), and Newfoundland and Labrador (St. John's, NL, Canada) through the Canadian Agricultural Partnership. We thank the participating FAPM veterinarians, participating FAPM senior veterinary students, SC, coaches, raters, and OSCE support personnel for their involvement and hard work. We also thank Brian Gromoff, University of Calgary, Calgary, Alberta, Canada, for his effort and skill training the standardized clients to portray the cases and to provide feedback to the participants during the small-group experiential training and on the SC feedback forms during the OSCE. The authors have not stated any conflicts of interest.

\section{REFERENCES}

Adams, C. L., and S. M. Kurtz. 2006. Building on existing models from human medical education to develop a communication curriculum in veterinary medicine. J. Vet. Med. Educ. 33:28-37. https:/ /doi.org/10.3138/jvme.33.1.28.

Adams, C. L., and S. M. Kurtz. 2012. Coaching and feedback: Enhancing communication teaching and learning in veterinary practice settings. J. Vet. Med. Educ. 39:217-228. https://doi.org/10.3138/ jvme.0512-038R.

Adams, C. L., and S. M. Kurtz. 2017. Skills for Communicating in Veterinary Medicine. Dewpoint Publishing.

Adams, C. L., and L. Ladner. 2004. Implementing a simulated client program: Bridging the gap between theory and practice. J. Vet. Med. Educ. 31:138-145. https://doi.org/10.3138/jvme.31.2.138.

American Animal Hospital Association. 2009. Compliance: Taking Quality Care to the Next Level: A Report of the 2009 AAHA Compliance Follow-Up Study. American Animal Hospital Association.

American Veterinary Medical Association. 2020. Council on Education (COE) Accreditation Policies and Procedures: Requirements. Accessed March 13, 2021. https://www.avma.org/education/ accreditation-policies-and-procedures-avma-council-education -coe/coe-accreditation-policies-and-procedures-requirements.

Ammentorp, J., S. Sabroe, P. Kofoed, and J. Mainz. 2007. The effect of training in communication skills on medical doctors' and nurses' self-efficacy: A randomized control trial. Patient Educ. Couns. 66:270-277. https://doi.org/10.1016/j.pec.2006.12.012.

Artemiou, E., C. L. Adams, K. G. Hecker, A. Vallevand, C. Violato, and J. B. Coe. 2014a. Standardised clients as assessors in a veterinary communication OSCE: A reliability and validity study. Vet. Rec. 175:509. https://doi.org/10.1136/vr.102633.

Artemiou, E., C. L. Adams, L. Toews, C. Violato, and J. B. Coe. 2014 b. Informing web-based communication curricula in veteri- 
nary education: A systematic review of web-based methods used for teaching and assessing clinical communication in medical education. J. Vet. Med. Educ. 41:44-54. https://doi.org/10.3138/jvme .0913-126R.

Artemiou, E., C. L. Adams, A. Vallevand, C. Violato, and K. G. Hecker. 2013. Measuring the effectiveness of small-group and web-based training methods in teaching clinical communication: A case comparison study. J. Vet. Med. Educ. 40:242-251. https://doi.org/10 .3138/jvme.0113-026R1.

Association of American Veterinary Medical Colleges. 2018. Competency-Based Veterinary Education: Part 1 - CBVE Framework. Association of American Veterinary Medical Colleges.

Barton, K., C. D. Cunningham, G. T. Jones, and P. Maharg. 2006. Valuing what clients think: Standardized clients and the assessment of communicative competence. Clin. Law Rev. 13:1-65.

Bauman, C. A., H. W. Barkema, J. Dubuc, G. P. Keefe, and D. F. Kelton. 2016. Identifying management and disease priorities of Canadian dairy industry stakeholders. J. Dairy Sci. 99:10194-10203. https://doi.org/10.3168/jds.2016-11057.

Berkhof, M., H. J. van Rijssen, A. J. M. Schellart, J. R. Anema, and A. J. van der Beek. 2011. Effective training strategies for teaching communication skills to physicians: An overview of systematic reviews. Patient Educ. Couns. 84:152-162. https://doi.org/10.1016/ j.pec.2010.06.010.

Cipolla, M., and A. Zecconi. 2015. Short communication: Study on veterinarian communication skills preferred and perceived by dairy farmers. Res. Vet. Sci. 99:60-62. https://doi.org/10.1016/j.rvsc .2015 .02 .004 .

Davis, M. H., G. G. Ponnamperuma, S. McAleer, and V. H. M. Dale. 2006. The objective structured clinical examination (OSCE) as a determinant of veterinary clinical skills. J. Vet. Med. Educ. 33:578-587. https://doi.org/10.3138/jvme.33.4.578.

Eekhout, I., H. C. W. de Vet, J. W. R. Twisk, J. P. L. Brand, M. R. de Boer, and M. W. Heymans. 2014. Missing data in a multiitem instrument were best handled by multiple imputation at the item score level. J. Clin. Epidemiol. 67:335-342. https://doi.org/ 10.1016/j.jclinepi.2013.09.009.

Goodwin, L. D. 2001. Interrater agreement and reliability. Meas. Phys. Educ. Exerc. Sci. 5:13-34. https://doi.org/10.1207/ S15327841MPEE0501_2.

Gulbrandsen, P., B. F. Jensen, A. Finset, and D. Blanch-Hartigan. 2013. Long-term effect of communication training on the relationship between physicians' self-efficacy and performance. Patient Educ. Couns. 91:180-185. https://doi.org/10.1016/j.pec.2012.11 .015 .

Hargie, O., M. Boohan, M. McCoy, and P. Murphy. 2010. Current trends in communication skills training in UK schools of medicine. Med. Teach. 32:385-391. https://doi.org/10.3109/01421590903394603.

Hecker, K. G., C. L. Adams, and J. B. Coe. 2012. Assessment of firstyear veterinary students' communication skills using an objective structured clinical examination: The importance of context. J. Vet. Med. Educ. 39:304-310. https://doi.org/10.3138/jvme.0312.022R.

Hodges, B. D. 2006. The objective structured clinical examination: Three decades of development. J. Vet. Med. Educ. 33:571-577. https://doi.org/10.3138/jvme.33.4.571.

Humphris, G. M. 2002. Communication skills knowledge, understanding and OSCE performance in medical trainees: A multivariate prospective study using structural equation modelling. Med. Educ. 36:842-852. https://doi.org/10.1046/j.1365-2923.2002.01295.x.

Jansen, J., C. D. M. Steuten, R. J. Renes, N. Aarts, and T. J. G. M. Lam. 2010. Debunking the myth of the hard-to-reach farmer: Effective communication on udder health. J. Dairy Sci. 93:12961306. https://doi.org/10.3168/jds.2009-2794.

Kleen, J. L., O. Atkinson, and J. P. T. M. Noordhuizen. 2011. Communication in production animal medicine: modelling a complex interaction with the example of dairy herd health medicine. Ir. Vet. J. 64:8. https://doi.org/10.1186/2046-0481-64-8.

Kurtz, S. 2006. Teaching and learning communication in veterinary medicine. J. Vet. Med. Educ. 33:11-19. https://doi.org/10.3138/ jvme.33.1.11.
Lam, T. J. G. M., J. Jansen, B. H. P. van den Borne, R. J. Renes, and H. Hogeveen. 2011. What veterinarians need to know about communication to optimise their role as advisors on udder health in dairy herds. N. Z. Vet. J. 59:8-15. https://doi.org/10.1080/ 00480169.2011.547163.

MacDonald-Phillips, K. A. 2021. Program outcomes of a voluntary Johne's disease control program with a focus on adherence to management plan recommendations and veterinary communication skills. PhD Thesis. Department of Health Management, Faculty of Veterinary Medicine, University of Prince Edward Island, Charlottetown, PEI.

MacDonald-Phillips, K. A., S. L. B. McKenna, D. H. Shaw, G. P. Keefe, J. VanLeeuwen, E. Artemiou, and C. L. Adams. 2021. Communication skills training and assessment of food animal production medicine veterinarians: a component of a voluntary Johne's disease control program - Supplemental file. University of Prince Edward Island, Robertson Library's Data Repository. https://data .upei.ca/islandora/object/researchdata\%3A680.

Mullan, B. A., and E. J. Kothe. 2010. Evaluating a nursing communication skills training course: The relationships between self-rated ability, satisfaction, and actual performance. Nurse Educ. Pract. 10:374-378. https://doi.org/10.1016/j.nepr.2010.05.007.

Petrovski, K. R., and M. McArthur. 2015. The art and science of consultations in bovine medicine: Use of modified Calgary-Cambridge guides. Maced. Vet. Rev. 38:137-147. https://doi.org/10.14432/j macvetrev.2015.05.044.

Radford, A., P. Stockley, J. Silverman, I. Taylor, R. Turner, C. Gray, L. Bush, M. Glyde, A. Healy, V. Dale, S. Kaney, C. Magrath, S. Marshall, S. May, B. McVey, C. Spencer, R. Sutton, J. Tandy, P. Watson, and A. Winter. 2006. Development, teaching, and evaluation of a consultation structure model for use in veterinary education. J. Vet. Med. Educ. 33:38-44. https://doi.org/10.3138/jvme .33.1.38.

Ritter, C., C. L. Adams, D. F. Kelton, and H. W. Barkema. 2018. Clinical communication patterns of veterinary practitioners during dairy herd health and production management farm visits. J. Dairy Sci. 101:10337-10350. https://doi.org/10.3168/jds.2018 $-14741$.

Ritter, C., C. L. Adams, D. F. Kelton, and H. W. Barkema. 2019. Factors associated with dairy farmers' satisfaction and preparedness to adopt recommendations after veterinary herd health visits. J. Dairy Sci. 102:4280-4293. https://doi.org/10.3168/jds.2018-15825.

Roter, D. L., R. Wexler, P. Naragon, B. Forrest, J. Dees, A. Almodovar, and J. Wood. 2012. The impact of patient and physician computer mediated communication skill training on reported communication and patient satisfaction. Patient Educ. Couns. 88:406413. https://doi.org/10.1016/j.pec.2012.06.020.

Shaw, J. R. 2019. Evaluation of communication skills training programs at North American veterinary medical training institutions. J. Am. Vet. Med. Assoc. 255:722-733. https://doi.org/10.2460/ javma.255.6.722.

Shaw, J. R., C. L. Adams, and B. N. Bonnett. 2004. What can veterinarians learn from studies of physician-patient communication about veterinarian-client-patient communication? J. Am. Vet. Med. Assoc. 224:676-684. https://doi.org/10.2460/javma.2004 .224 .676 .

Shaw, J. R., C. L. Adams, B. N. Bonnett, S. Larson, and D. L. Roter. 2012. Veterinarian satisfaction with companion animal visits. J. Am. Vet. Med. Assoc. 240:832-841. https://doi.org/10.2460/ javma.240.7.832.

Shaw, J. R., G. E. Barley, A. E. Hill, S. Larson, and D. L. Roter. 2010. Communication skills education onsite in a veterinary practice. Patient Educ. Couns. 80:337-344. https://doi.org/10.1016/j.pec .2010.06.012.

Silverman, J., S. M. Kurtz, and J. Draper. 2013. Skills for Communicating with Patients. Third Edition. CRC Press, Taylor \& Francis Group.

Sorge, U., D. Kelton, K. Lissemore, A. Godkin, S. Hendrick, and S. Wells. 2010a. Attitudes of Canadian dairy farmers toward a volun- 
tary Johne's disease control program. J. Dairy Sci. 93:1491-1499. https://doi.org/10.3168/jds.2009-2447.

Sorge, U. S., J. Mount, D. F. Kelton, and A. Godkin. 2010b. Veterinarians' perspective on a voluntary Johne's disease prevention program in Ontario and western Canada. Can. Vet. J. 51:403-405.

van Ginkel, J. R., K. Sijtsma, L. A. van der Ark, and J. K. Vermunt. 2010. Incidence of missing item scores in personality measurement, and simple item-score imputation. Methodology 6:17-30. https:// doi.org/10.1027/1614-2241/a000003.

Wolf, R., H. W. Barkema, J. De Buck, and K. Orsel. 2015. Factors affecting management changes on farms participating in a Johne's disease control program. J. Dairy Sci. 98:7784-7796. https://doi .org/10.3168/jds.2015-9610.

World Organization for Animal Health. 2012. OIE Recommendations on the Competencies of Graduating Veterinarians ('Day 1 Graduates') to Assure National Veterinary Services of Quality. OIE.

Wraight, M. D., J. McNeil, D. S. Beggs, R. K. Greenall, T. B. Humphris, R. J. Irwin, S. P. Jagoe, A. Jemmeson, W. F. Morgan,
P. Brightling, G. A. Anderson, and P. D. Mansell. 2000. Compliance of Victorian dairy farmers with current calf rearing recommendations for control of Johne's disease. Vet. Microbiol. 77:429442. https://doi.org/10.1016/S0378-1135(00)00328-X.

\section{ORCIDS}

Karen A. MacDonald-Phillips (- https://orcid.org/0000-0001-5218 $-564 \mathrm{X}$

Shawn L. B. McKenna (ํ) https://orcid.org/0000-0002-9699-1464

Greg P. Keefe @ https://orcid.org/0000-0003-2356-7344

John VanLeeuwen (1) https://orcid.org/0000-0003-3340-0337

Cindy L. Adams ๑ https://orcid.org/0000-0003-2390-4347 